\title{
Morphology transformation in pairs of galaxies - the local sample
}

\author{
S. Junqueira ${ }^{1}$, D.F. de Mello ${ }^{1, \star}$, and L. Infante ${ }^{2}$ \\ 1 Observatório Nacional - C. Postal 23002, Rio de Janeiro, 20921-400, Brazil \\ 2 Departamento de Astronomía y Astrofísica, P. Universidad Católica de Chile, Casilla 104, Santiago 22, Chile
}

Received April 1; accepted September 24, 1997

\begin{abstract}
We present photometric analysis of a local sample of 14 isolated pairs of galaxies. The photometric properties analyzed in the local pairs are: colors, morphology, tidal effects and activity. We verify that close pairs have an excess of early-type galaxies and many elliptical galaxies in this pairs are, in fact, lenticular galaxies. Many late-pairs in our sample show strong tidal damage and blue star formation regions. We conclude that pairs of different morphologies may have passed through different evolution processes which violently transformed their morphology. Pairs with at least one early-type component may be descendents of groups of galaxies. However, late-type pairs are probably long-lived showing clearly signs of interaction. Some of them could be seen as an early stage of mergers. These photometric databases will be used for future comparison with more distant pairs in order to study galaxy evolution.
\end{abstract}

Key words: galaxies: evolution - galaxies: fundamental parameters - galaxies: interactions galaxies: photometry

\section{Introduction}

Since Hubble $(1926,1936)$ categorized galaxies according to their shapes, galaxy morphology has been used as a fundamental parameter in studies of galaxy formation and evolutionary processes. However, many galaxies have unusual morphologies and do not belong to any Hubble type. Most of these peculiar galaxies are interacting systems and had their forms distorted by tidal forces. Therefore, interactions between galaxies are a major evolution mechanism which transforms galaxy morphology during their evolution. The so-called Toomre's sequence (Toomre 1977) is a perfect example of what happens to

Send offprint requests to: selma@on.br

* Present address: STScI, Baltimore MD 21218, U.S.A. galaxy morphology during different stages of interaction. Numerical simulations of the encounter between galaxies has established that gravitational interaction can not only originate tidal damage to spiral structures but also merge two disk galaxies in one final product which resembles an elliptical galaxy (Toomre \& Toomre 1972, see Barnes \& Hernquist 1992 for a review). In order to establish whether an encounter will originate a merger or not it is necessary to determine the physical properties and dynamics of the interaction. In principle, all systems made of galaxies with comparable mass and which are in elliptical bound orbits end as a merger but not necessarily within the Hubble time (Binney \& Tremaine 1987; Junqueira \& de Freitas Pacheco 1994).

If mergers are frequent in galaxy evolution we should be able to find mergers in progress such as NGC 7252 and/or traces of the effect produced by this phenomenon. For instance, the most commonly associated features invoked by merger theory are bars, isophotes twisting, color gradient, shells, inner structures, dust, starbursts, AGN's, etc (Schweizer 1983; Noguchi 1988; Bender et al. 1989; Forbes \& Thomson 1992). However, to what degree are the galaxy properties influenced by interactions with its neighbors is still an open issue. For instance, interaction involving at least one gas-rich galaxy may result in activity which may range from global starbusts to nuclear ones and to AGN's, however it is unclear whether there is a transition between these three features (Fritze-v. Alvensleben 1996; Keel 1996).

One of the most striking evidences for galaxy evolution is the faint blue galaxies excess at $z \geq 0.3$, i.e. the Buchter \& Oemler effect (Butcher \& Oemler 1978, 1984). In order to explain this effect, galaxy evolution models need a mechanism for creating disturbed galaxies with enhanced star formation and explain why they occur more frequently at this redshift (Moore et al. 1996). Moreover, they have to address the question of morphology evolution and an identification of the remnants of the distorted blue galaxies in the local Universe. These issues have been the focus of major research efforts, largely triggered by the 
Hubble Deep Field observations (Williams et al. 1996) and the $10 \mathrm{~m}$ class telescope data which are becoming available now. However, despite the efforts made so far, fundamental questions, such as when, where and how galaxies form and evolve remain controversial. For instance, in hierarchical models for galaxy formation an individual galaxy may pass through various phases of disk or spheroid during its lifetime (Baugh et al. 1996). The authors claim that about $50 \%$ of all ellipticals (but only about $15 \%$ of spirals) would have undergone a major merger during the redshift interval $0.0 \leq z \leq 0.5$. However, the high degree of uniformity in ellipticals star formation history indicates that these objects must have formed at $z \geq 2$ (Bower et al. 1992).

An essential step towards answering these questions is understanding the properties of interacting galaxies in the local universe and comparing it with the more distant objects. We have started a long-term project focusing on these issues. Our first step was to select a sample of faint interacting galaxies in pairs and groups. By faint we mean galaxies that are within $19<m_{R}<22$ and by interacting we mean angular separations of $2^{\prime \prime}<\theta<6^{\prime \prime}$ (see Infante et al. 1996; de Mello et al. 1997a,b for more details). Among the more interesting results we find is the excess of faint pairs at intermediate redshift $(z \approx 0.35)$.

In this paper we present a continuation of our previous work. We present a database of nearby pairs of galaxies that will be used in the future to compare with pairs at intermediate redshifts.

\section{The sample}

\subsection{Binary galaxies}

The ideal sites to study galaxy encounters are pairs and groups of galaxies. The pioneers in the studies of pairs of galaxies are Holmberg $(1937,1958)$ and Page $(1952,1961)$ and more recently Karachentsev (1972). Major effort has been focused into assembly complete samples of isolated pairs of galaxies for the Southern Hemisphere (Reduzzi \& Rampazzo 1995; Soares et al. 1995; see Sulentic 1992 for a review on the subject). Several studies have analyzed the effect of interaction in binary galaxies, e.g. Rampazzo \& Sulentic (1992); Rampazzo et al. (1995); de Mello et al. (1996); Marquez \& Moles 1996; Reduzzi \& Rampazzo (1996), and de Souza et al. (1997).

One simple approach in studying tidal effects is to analyze galaxy morphology in binary galaxies. A standard morphological classification of binaries is based on their components morphology. For instance, early-type binaries, usually called EE, are formed by two early-type galaxies and late-type binaries (SS) by two spiral galaxies. Mixed morphology binaries, ES, are formed by an early and a late-type galaxy. The observed fraction of the three types is 0.60 for SS, 0.30 for for ES and 0.10 for EE (Sulentic 1992). The existence of many isolated mixed binaries raises problems for models of galaxy for- mation that envision local environment as a major determinant of morphology. However, there are a few evidences, like morphological distortion in the late-type galaxy and young stellar population in the early-type showing that mixed binaries are physically interacting (de Mello et al. 1996; Rampazzo \& Sulentic 1992). Therefore, if mergers of spiral galaxies are the mechanism for the formation of ellipticals then binaries containing an elliptical would be the product of interaction between at least 3 galaxies. To understand whether galaxy transformation is a common phenomenon in galaxy evolution more studies of galaxy morphologies in interacting systems are needed. In this work we present the results of such analysis for a sample of binary galaxies.

\subsection{Selection criteria}

One of the most important goals of this study is to build a photometric and spectroscopic database in order to study interacting galaxy evolution. For this, the bright and faint pairs should constitute a carefully matched sample with projected separations such that the objects would be expected to merge in much less than a Hubble time.

The faint $\left(19<m_{R}<22\right)$ sample was selected from automatic compiled catalogs of existing (de Mello et al. 1997a,b) CTIO $4 \mathrm{~m}$ images at the equator; the bright sample $\left(16<m_{V}<18\right)$ was selected from the catalog of isolated binary galaxies (CPG) compiled by Karachentsev (1972), which is the best available catalog that meets the local control sample requirements.

Pairs of faint galaxies (separation $\Delta \theta$ ) were chosen such that $\theta_{\min }<\Delta \theta<\theta_{\max }$. Here $\theta_{\min }$ is the minimum separation at which pairs can be reliably separated (nominally $\left.2^{\prime \prime}\right) ; \theta_{\max }$ corresponds to some physical separation, $r_{\mathrm{p}}$, chosen so that: (i) physical pairs are doomed to merge in $<10^{9} \mathrm{yr}$ (on the basis of both empirical studies and conventional dynamics); and (ii) most pairs in the faint sample are physically associated. These conditions are satisfied by $r_{\mathrm{p}} \simeq 20 \mathrm{kpc}$, which corresponds to $\sim 6^{\prime \prime}$ at $z=0.35$.

What fraction of the faint pairs is expected to be physical pairs and merge? Infante et al. (1996) have calculated the angular correlation function, $w(\theta)=1.75$ for pairs at $m_{R}<21.5$ and $\theta<6^{\prime \prime}$. It follows that $\approx 64 \%$ of faint pairs will be physically associated. Furthermore, a cut in $\Delta v$ (say $\Delta v<1000 \mathrm{~km} \mathrm{~s}^{-1}$ ) should isolate most of the physical pairs. Following Carlberg et al. (1994) the fraction of galaxies that will merge is $70 \%$. Thus, it is expected that $\approx 45 \%$ of galaxies in pairs will eventually merge.

The two samples should be affected by identical selection effects. The CPG contains 603 binaries brighter than $m_{\mathrm{pg}}=15.7$, is the most complete catalog of binaries and has complete redshift information. In order to match the properties of $z=0.35$ pairs we looked at all Karachentsev pairs with characteristics similar to what we expected for pairs at high $z$. We have selected 
all pairs with projected linear separation $\leq 23 \mathrm{~h}^{-1} \mathrm{kpc}$ $\left(\mathrm{h}^{-1}=100 / H_{0} \mathrm{~km} \mathrm{~s}^{-1} \mathrm{Mpc}^{-1}\right)$ and radial velocity difference $\leq 420 \mathrm{~km} \mathrm{~s}^{-1}$.

\section{Data reduction and photometric calibrations}

$B, V$ and $I$ images were taken at the Cassegrain focus of the $2.2 \mathrm{~m} \mathrm{MPI/ESO} \mathrm{telescope} \mathrm{with} \mathrm{EFOSC2,} \mathrm{CCD19}$ $\left(1024 \times 1024\right.$ with a scale of $0.336^{\prime \prime}$ pixel $\left.^{-1}\right)$ during 3 photometric nights in la Silla, Chile, in October 1994. Typical exposure times were $900 \mathrm{~s}$ in $B, 300 \mathrm{~s}$ in $V$ and $600 \mathrm{~s}$ in I. Observational conditions were photometric with an average seeing of $1.2^{\prime \prime}$.

Initial reductions followed standard procedures, and were performed with IRAF. The bias level was subtracted from each image which were then divided by the averaged flat field exposure for the night. In Figs. 1 and 2 we show the grey-scale $V$ images of the 14 pairs in our sample.

Table 1. Calibration coefficients

\begin{tabular}{cccc}
\hline Filter & $m_{0}$ & $m_{x}$ & $\mathrm{rms}$ \\
\hline & & & \\
$B$ & -0.0027 & 0.2402 & 0.006 \\
$V$ & -0.0026 & 0.1531 & 0.012 \\
$I$ & -0.0027 & 0.0649 & 0.009 \\
& & & \\
\hline
\end{tabular}

Standard stars from Landolt's list (1992), MarkA, $\mathrm{T}$ Phe and SAO 98 were observed for extinction and calibration purposes. To calibrate the images we used the following equations:

$$
\begin{aligned}
& b-B=A_{1}+A_{2} X+A_{3}(B-V) \\
& v-V=C_{1}+C_{2} X+C_{3}(B-V) \\
& i-I=D_{1}+D_{2} X+D_{3}(V-I) \\
& b-v=E_{1}+E_{2} X+E_{3}(B-V) \\
& v-i=F_{1}+F_{2} X+F_{3}(V-I)
\end{aligned}
$$

where $B, V$ and $I$ are the standard magnitudes, $b, v$ and $i$ are the instrumental magnitudes, $X$ is the airmass of the observation and the coefficients $A, C, D, E$ and $F$ are the transformation coefficients. $B$ was obtained from the relation $B=b+m_{0}-m_{x} X$, where $m_{0}$ is $m_{0}=-A_{1}-$ $A_{3}\left((b-v)-E_{1}\right) / E_{3}$ and $m_{x}=\left(A_{2}-A_{3} E_{2} / E_{3}\right)$. Similar relations were derived for $V$ and $I$.
In Table 1 we present the values of the extinction and color coefficients, $m_{x}$ and $m_{0}$, and the rms of the fit obtained for each filter. Mean extinction coefficients provided by ESO were used for comparison.

Two-dimensional surface photometry was accomplished using the last version of GALPHOT image analysis package developed and kindly provided by W. Freudling. This package runs in the IRAF environment and uses the ellipse-fitting routine available in the ISOPHOTE package of STSDAS following the method described by Jedrzejewski (1987). Prior to ellipse fitting, the sky background is removed and field stars and other features are automatically masked outside the outer isophotes of the galaxy.

Stars overlapping galaxies were carefully removed by extracting elliptical models which were substituted using an interpolation across the region. For pairs with overlapping galaxies such as CPG018, CPG083, CPG091, CPG099, CPG547, CPG577, we used the following procedure: first we built a model of the pair component $\mathbf{a}$, called model $a$ using the ELLIPSE and BMODEL. Then we subtracted this model from the original image, creating an image 2. From image 2 we built a model for galaxy $\mathbf{b}$, called model $b$. Model $b$ was subtracted from the original image, creating image 3, which was then used to do photometry of galaxy a (see Fig. 3a for an example of image 3). With the same procedure we have created images 4, 5 which was used to do photometry of galaxy $\mathbf{b}$ (see Fig. 3b for an example of image 5). In order to test this method we have applied it to synthesized images. Good results were obtained when the overlapped area was less than $10 \%$ of the total area. The goal of this procedure was to recover the overlapping external regions of the galaxies. For the cases where there were remaining features in the center of the subtracted galaxies, we masked these features before doing photometry of the companions. Photometry of CPG018 and CPG083 should be used with caution since they present a common envelope.

The radial surface luminosity profiles of the galaxies were fitted by an exponential law of the form

$\Sigma(r)=\Sigma_{o} \exp (-r / \alpha)^{\beta}$

where $\Sigma(r)$ is the surface luminosity density (in $L_{\odot} \mathrm{pc}^{-2}$ ) corresponding to the surface brightness, $\mu$ (in mag $\operatorname{arcsec}^{-2}$ ), and $r$ is the major axis radius. The fit parameters are then the central surface brightness, $\Sigma_{0}\left(\mu_{0}\right)$, and the exponential scale length, $\alpha$. The value of $\beta$ will depend on the morphological type of the galaxy, being 1 for spiral disks, and 1/4 for ellipticals or spiral bulges (de Vaucouleurs 1948). The following relations were used in order to fit the surface brightness versus radius and obtain the fit parameters:

$\mu_{\text {disk }}=\mu_{0}+1.0857(r / \alpha)$ 
CPG018

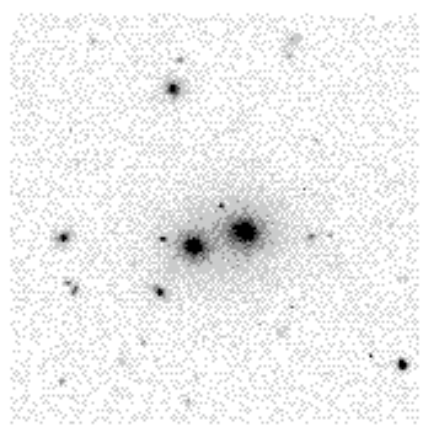

CPG091

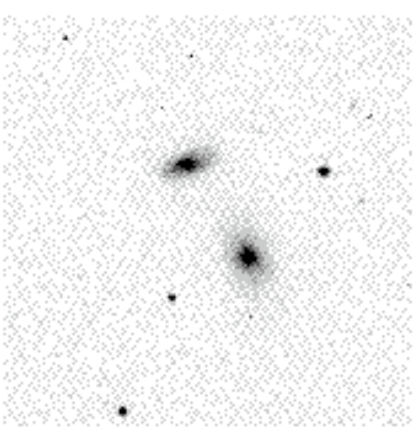

CPG547

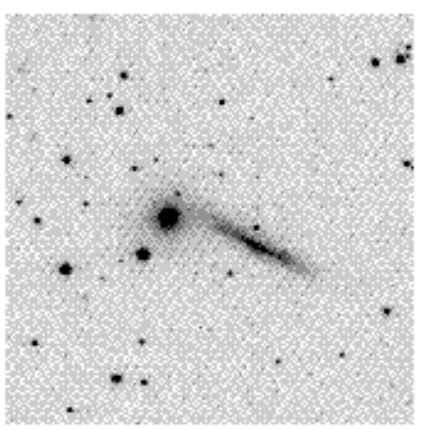

CPG069

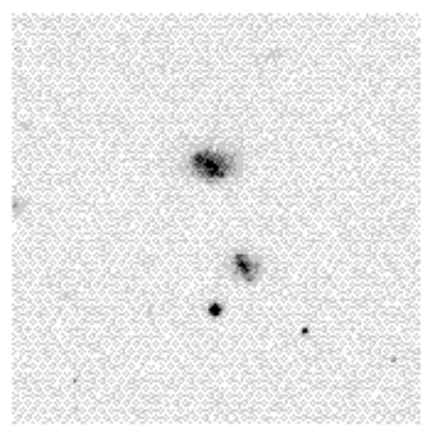

CPG099

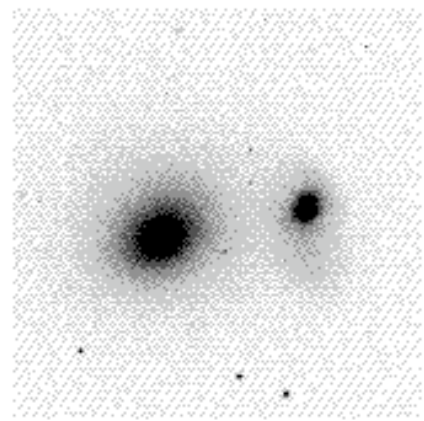

CPG548

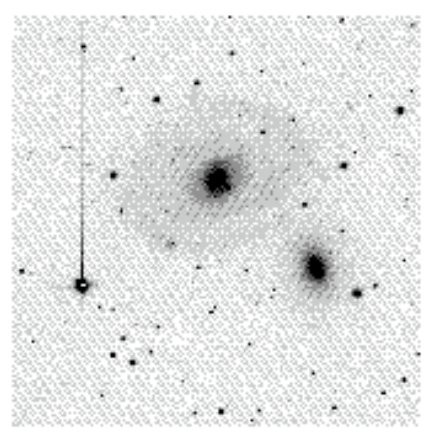

\section{CPG083}

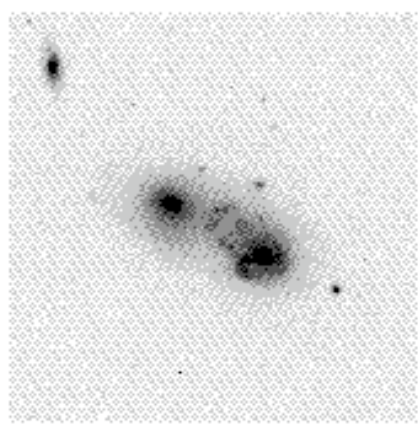

CPG545

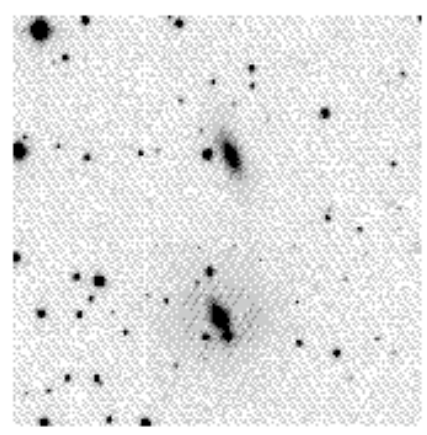

CPG551

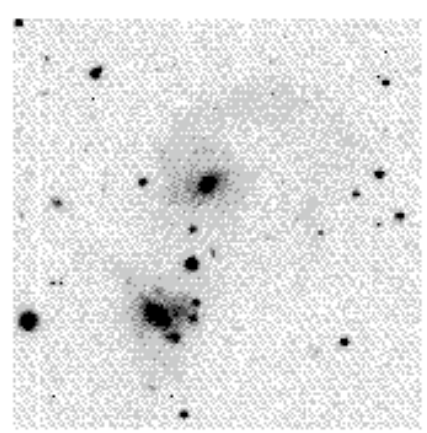

Fig. 1. Grey-scale $V$ images of 9 Karachentsev's pairs of galaxies in our sample. North is to the top and East to the left. For the pair CPG548 the size of the image is $5.8^{\prime} \times 5.8^{\prime}$. For the other 8 images the size is $2.9^{\prime} \times 2.9^{\prime}$

$\mu_{\text {bulge }}=\mu_{\mathrm{e}}+8.33\left(\left(r / r_{\mathrm{e}}\right)^{1 / 4}-1\right)$

where $\alpha$ is the disk scale length and $\mu_{0}$ is the disk central surface brightness, $r_{\mathrm{e}}$ and $\mu_{\mathrm{e}}$ are the effective radius and the surface brightness at $r_{\mathrm{e}}$ radius and surface brightness at $r_{\mathrm{e}}$. We present the radial surface brightness distributions and the exponential fits in Figs. 4 and 5.
The total magnitudes were calculated by extrapolating the luminosity profiles to infinity. For several galaxies, bulge and disk components were deconvolved from the surface brightness profile following a standard interactive procedure (Boroson 1981; Schombert \& Bothun 1987; Marquez \& Moles 1996). First, we have fitted the outer 
CPG575

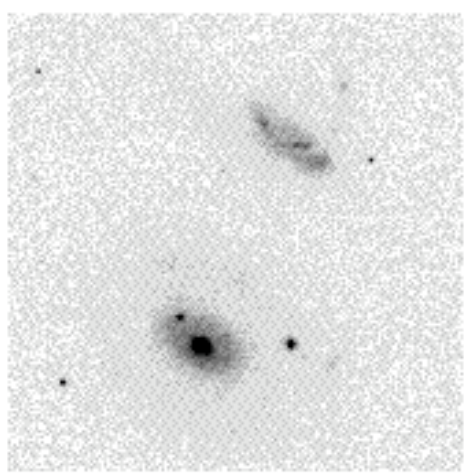

CPG577 CPG580
CPG583

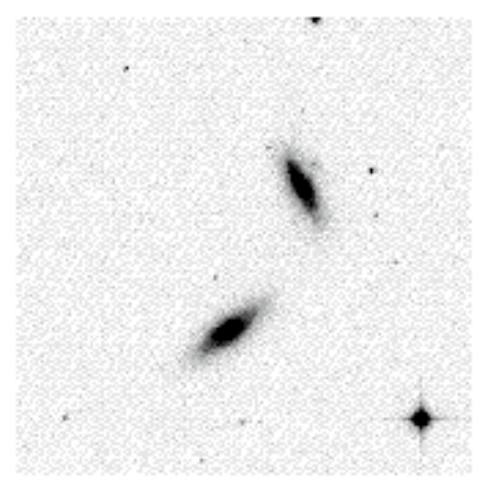

CPG587

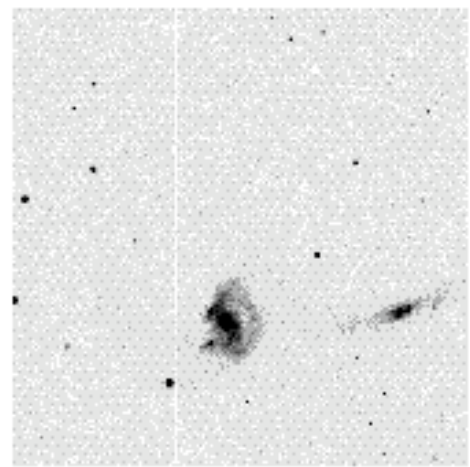

Fig. 2. Grey-scale $V$ images of 5 Karachentsev's pairs of galaxies in our sample. North is to the top and, East to the left. For the pair CPG587 the size of the image is $5.8^{\prime} \times 5.8^{\prime}$. For the other 4 images the size is $2.9^{\prime} \times 2.9^{\prime}$

regions of the surface brightness profile using an exponential law. We have subtracted the fitted disk profile from the total profile obtaining the bulge profile. Then, the bulge profile was fitted using an $r^{1 / 4}$ law. Finally, the fitted profiles (disk and bulge) were summed and the total fitted profile was compared with the total observed profile. After some iteractions the method converges and we obtain the lowest $\chi^{2}$ fit. The total magnitudes correspond to the lowest $\chi^{2}$. The major uncertainty on the total (asymptotic) magnitude values depends on the error on the surface brightness $(\mu)$ calculated at the outermost point reached by the profile determination (due to the uncertainty on the sky level) and on the error due to the extrapolation procedure. Taking into account all contributions, the total uncertainties are $0.18,0.13$ and 0.12 for $B, V$ and $I$, respectively. The total magnitudes $B, V$ and $I$, were corrected for inclination following Burstein (1979); Binggeli (1980) and Franx et al. (1991) for the ellipticals.
For the spirals we have assumed round disks. We have also corrected for galactic extinction using Burstein \& Heiles (1984) sample. For the galaxies that are not in their sample we have followed the method in RC2 (de Vaucouleurs et al. 1976).

In order to check the quality of our photometry we have compared our magnitudes with $m_{B}$ in the RC3 (de Vaucouleurs et al. 1991) and with other papers in the literature, specially with Marquez \& Moles (1994 and 1996, MM hereafter) values since our magnitude determination followed similar procedure. In Fig. 6 we plotted the values of our $B$ magnitudes before corrections (galactic extinction correction and inclination correction) and $m_{B}$ from RC3. CPG575b was already found as discrepant by MM and our result is closer to MM value than to RC3 (RC3 $m_{B}=15.08, \mathrm{MM} m_{B}=14.67$, our value $\left.m_{B}=14.14\right)$. CPG580a is discrepant in relation to RC3 by 0.86 magnitudes, but that could be due to presence of a 
a

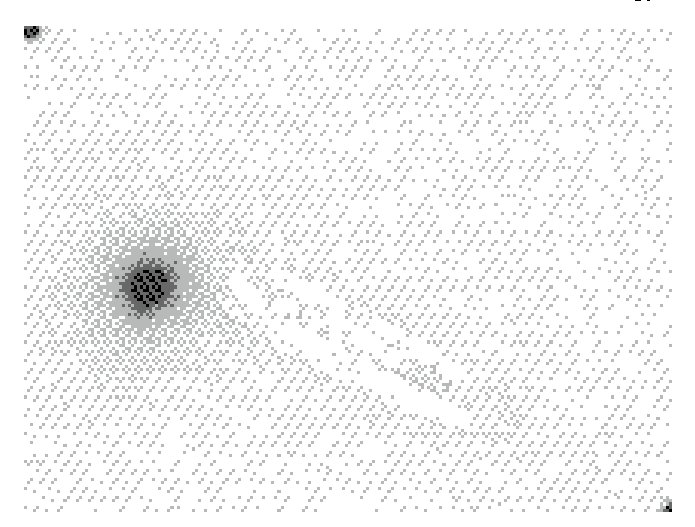

$b$

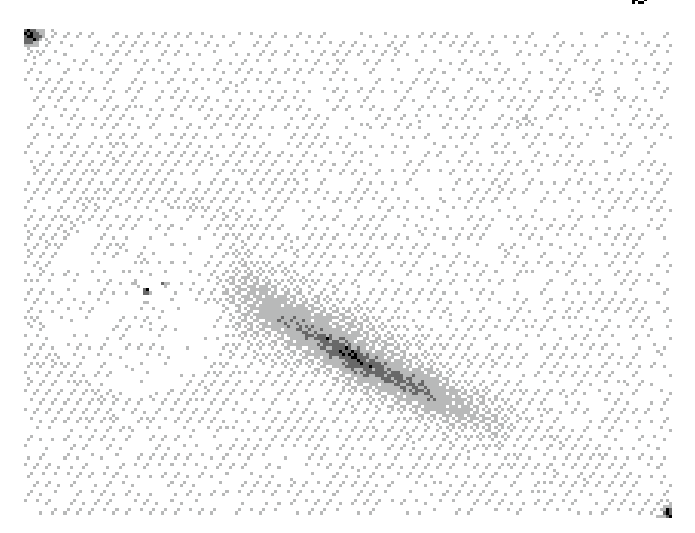

Fig. 3. a) Image of CPG547 after the model subtraction of galaxy b; b) Image of CPG547 after the model subtraction of galaxy a)

warped disk which makes the photometric decomposition not straightforward. The other two discrepant galaxies are CPG587a and $\mathrm{b}$. The distortion on the disks could also be responsible for this large difference in magnitudes $(0.83$ for CPG587a and 2.00 for CPG587b), however there is no additional magnitude determination in the literature for comparison. The other six galaxies that we have in common with RC3 have differences in magnitude $\leq 0.23$. CPG575a, for instance, is only 0.01 fainter in our determination than in RC3 and agrees with MM within 0.11 magnitudes, when no corrections are applied.

A few galaxies in our sample were studied by other authors. Reshetnikov et al. (1993) has done photometry of
CPG099, CPG587 and CPG575 however, we can not compare directly the total magnitude values because they have used another photometric system (the $R$ Cousins system). Rampazzo et al. (1995) studied CPG548 but they did not provide total magnitude values, only surface brightness profiles. They have remarked that the photometric profile of CPG548b (NGC 6964) follows an $r^{1 / 4}$ law, indicating that this object is a bona fide elliptical which is in agreement with our results for this galaxy. Heraudeau \& Simien (1996) have done the photometry of CPG548a galaxy extrapolating the brightness distribution by an exponential function. They obtained total magnitude values equal to 12.06 and 11.24 for $V$ and $I$ bands, respectively. Our total magnitude values for this object are different from these values maybe due to the differences in the decomposition method and the extrapolation procedure.

\section{Data analysis}

Table 2 lists the data for 14 Karachentsev's pairs observed. Column (1) gives the identification in Karachentsev's catalog of isolated pairs, Col. (2) gives other identifications, Col. (3) gives the pair position (1950.0). Columns (4) to (6) give the integrated magnitudes in $B, V, I$ calculated as described in the previous section. (7) and (8) give the colors $B-V$ and $V-I$. Column (9) gives the morphology of each galaxy obtained from NED ${ }^{1}(\rightarrow$ gives the new morphology found from the radial profiles). Column (10) gives the radial velocities $\left(\mathrm{km} \mathrm{s}^{-1}\right.$ obtained from NED).

\subsection{Morphological properties}

CPG018 is a very interesting EE pair with a common envelope. Elliptical models were fitted and surface brightness profiles follow an $r^{1 / 4}$ law, however the ellipticity of the isophotal contours vary from 0 to 0.4 outside 10 arcsec.

CPG069 is a late-type pair with the two galaxies showing tidal effects in their shapes. CPG069a is a warm IRAS galaxy with flux ratio $F_{60} / F_{30}=2.86$ and its spectra is typical of an HII region galaxy (Kailey \& Lebofsky 1988). CPG069a surface brightness profile in the central regions are not well fitted.

CPG083 is a strongly interacting mixed pair also known as Arp 118 or VV331. Both galaxies, NGC 1143(E) and NGC 1144 (S), show starburst activities and signs of tidal distortion. The spiral galaxy presents a knotty ring with a peculiar structure which can be interpreted as the product of an interaction of two disk galaxies (Hippelein 1989). From the surface brightness profile it is clear that NGC 1143 is in fact a lenticular galaxy.

CPG091 is a mixed pair with moderate signs of interaction. The elliptical galaxy presents a strong dust

\footnotetext{
1 The NASA/IPAC Extragalactic Database, NED, is operated by the Jet Propulsion Laboratory, California Institute of Technology, under contract with the National Aeronautics and Space Administration.
} 

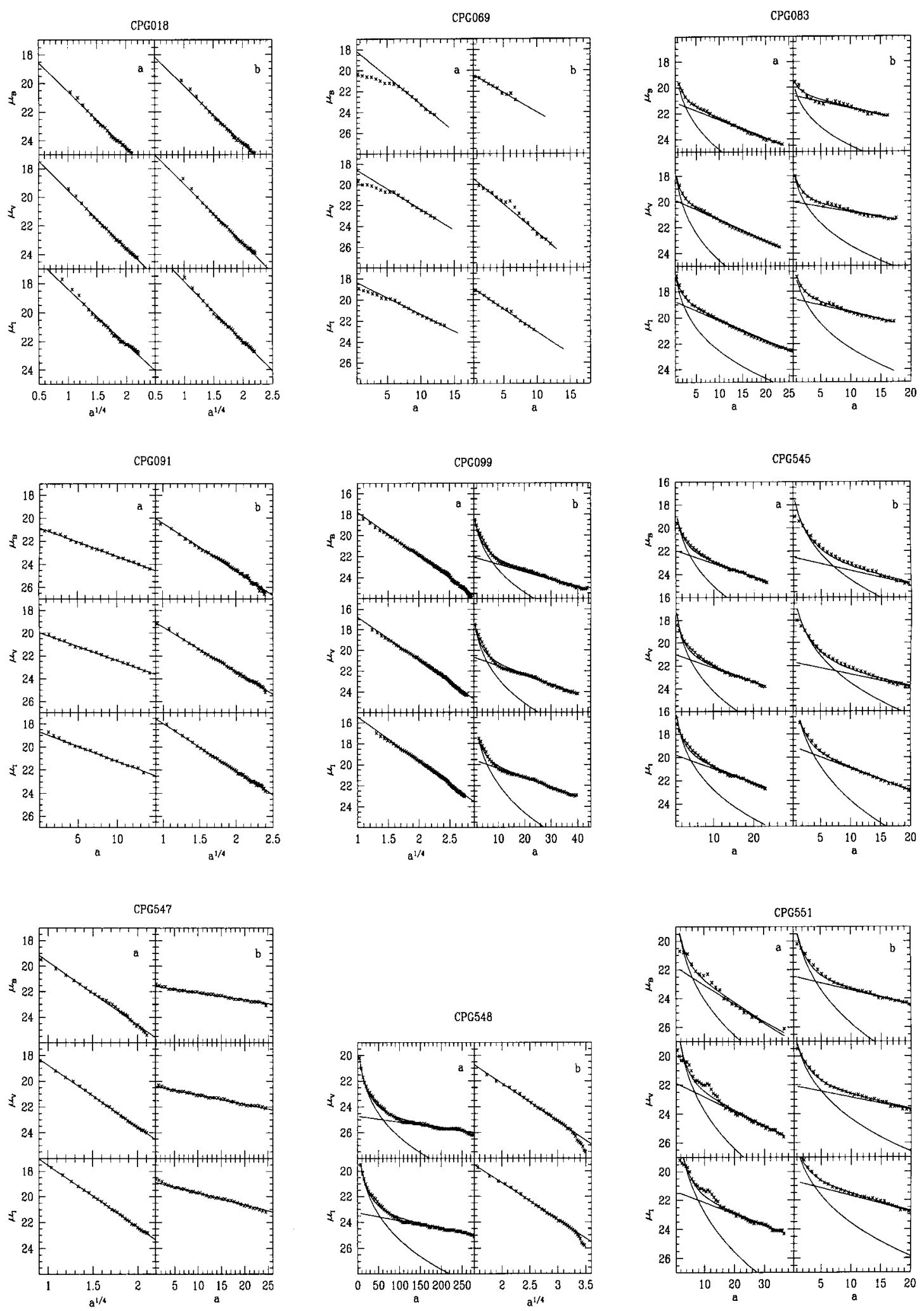

Fig. 4. Brightness profiles $\left(\mu_{B}, \mu_{V}\right.$ and $\left.\mu_{I}\right)$ and exponential fits (solid lines), in units mag/arcsec ${ }^{2}$, as a function of the semi-major axis $\left(a\right.$ or $a^{1 / 4}$ ) in units of arcsec, for both galaxies of 9 pairs of our sample in $B, V, I$ filters. a) and b) on the top indicate the members of the pairs 

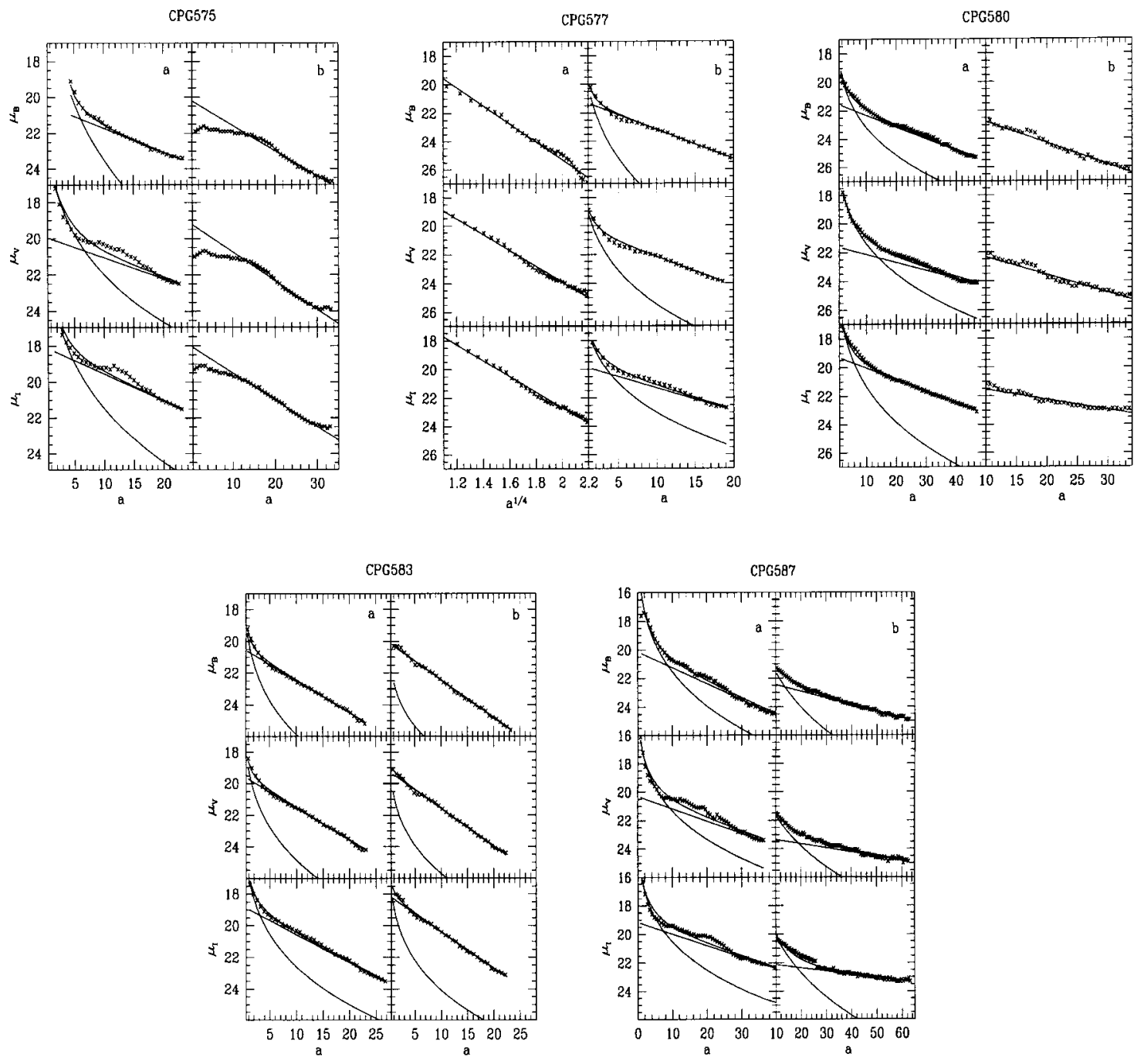

Fig. 5. The same as in Fig. 4 for five other pairs

lane and its surface brightness profile follows an $r^{1 / 4}$ law. The ellipticity of the isophotal contours is $\approx 0.4$ outside 10 arcsec.

CPG099 is an early-type pair strongly interacting. The two early-type galaxies (NGC 1587/88) show isophotal distortion and asymmetric light distribution. See Borne \& Hoessel (1988) for a simulation of this pair. From the surface brightness profile it is clear that NGC 1588 is in fact a lenticular galaxy. For NGC 1587 the ellipticity of the isophotal contours is $\approx 0.3$ outside 15 arcsec.

CPG545 is a late-type pair composed by a barred spiral and an Sb galaxy. Photometry of this pair is affected by bright stars in the field.

CPG547 is a mixed pair in close contact. The barred spiral galaxy is edge-on and overlaps the elliptical (Keel 1993). The surface brightness profiles of the elliptical galaxy follow an $r^{1 / 4}$ law. The ellipticity of the isophotal contours is quite irregular varying from $\approx 0$ to 0.2 outside 10 arcsec.

CPG548 is a mixed pair. The spiral galaxy has two long symmetric arms and a weak central bar and the elliptical has an elongated shape. The surface brightness profiles follow an $r^{1 / 4}$ law. Rampazzo et al. (1995) studied its kinematics and found signs of interaction. The ellipticity of the isophotal contours of NGC 6964 is $\approx 0.3$ outside 20 arcsec.

CPG551 is a late-type pair strongly disturbed by tidal effects.

CPG575 is a late-type pair of galaxy with one galaxy (IC 5283) very disturbed by tidal effects and the other one (NGC 7469) with an outer and an inner ring (Buta \& Crocker 1993; Marquez \& Moles 1994). Structures can be seen in their surface brightness profiles. Seyfert activity is present (Marquez \& Moles 1994). 
Table 2. Data

\begin{tabular}{|c|c|c|c|c|c|c|c|c|c|}
\hline Name & Id. & $\mathrm{RA}^{\mathrm{hm} \mathrm{s}} \mathrm{DEC}^{\circ \prime}$ & $B$ & $V$ & $I$ & $B-V$ & $V-I$ & Morph. & $V_{\mathrm{r}}$ \\
\hline CPG018a & UGC 00496 & $004554+0105.0$ & 15.11 & 14.15 & 12.71 & 0.96 & 1.44 & $\mathrm{E}$ & 18648 \\
\hline CPG018b & UGC 00496 & $004554+0105.0$ & 14.78 & 13.80 & 12.57 & 0.98 & 1.23 & $\mathrm{E}$ & 19003 \\
\hline CPG069a & IC0233 & $022903+0235.5$ & 14.24 & 14.15 & 13.44 & 0.09 & 0.71 & dIn & 8266 \\
\hline CPG069b & CGCG388-036 & $022903+0235.5$ & 15.82 & 15.59 & 14.63 & 0.23 & 0.96 & $\mathrm{Sab}$ & 8303 \\
\hline CPG083a & NGC1143 & $0252 \quad 39-0023.0$ & 14.20 & 13.29 & 12.03 & 0.91 & 1.26 & $\mathrm{E} \rightarrow \mathrm{S} 0$ & 8514 \\
\hline CPG083b & NGC1144 & $025239-0023.0$ & 13.23 & 12.14 & 11.20 & 1.14 & 0.94 & Sring & 8714 \\
\hline CPG091a & CGCG391-011 & $033245-0123.5$ & 15.26 & 14.55 & 13.35 & 0.71 & 1.20 & $\mathrm{Sa}$ & 10674 \\
\hline CPG091b & CGCG391-012 & $033245-0123.5$ & 14.82 & 13.78 & 12.47 & 1.04 & 1.31 & Edust & 11086 \\
\hline CPG099a & NGC1587 & $042806+0033.0$ & 12.29 & 11.50 & 10.25 & 0.79 & 1.25 & $\mathrm{E}$ & 3800 \\
\hline CPG099b & NGC1588 & $042806+0033.0$ & 13.35 & 12.46 & 11.11 & 0.89 & 1.35 & Epec $\rightarrow$ S0 & 3495 \\
\hline CPG545a & UGC11567 & $202542+0019.5$ & 14.08 & 13.12 & 11.94 & 0.96 & 1.18 & $\mathrm{SB} ?$ & 5762 \\
\hline CPG545b & CGCG373-015 & $202542+0019.5$ & 13.85 & 12.69 & 11.19 & 1.16 & 1.50 & $\mathrm{Sb}$ & 5732 \\
\hline CPG547a & UGC11593 & $203212+0748.0$ & 14.68 & 14.11 & 12.90 & 0.57 & 1.21 & $\mathrm{E}$ & 7856 \\
\hline CPG547b & UGC11593 & $203212+0748.0$ & 12.67 & 12.48 & 11.45 & 0.19 & 1.03 & $\mathrm{Sc}$ & 7887 \\
\hline CPG548a & NGC6962 & $204451+0007.5$ & & 11.07 & 10.58 & & 0.48 & $\mathrm{SAB}(\mathrm{r}) \mathrm{ab}$ & 4419 \\
\hline CPG548b & NGC6964 & $204451+0007.5$ & & 12.20 & 10.88 & & 1.32 & $\mathrm{E}$ & 4032 \\
\hline CPG551a & UGC11657 & $2057 \quad 12-0204.0$ & 14.00 & 13.49 & 12.40 & 0.51 & 1.09 & $\mathrm{Pec}$ & 6033 \\
\hline CPG551b & UGC11658 & $\begin{array}{lllll}20 & 57 & 12 & -02 & 04.0\end{array}$ & 14.50 & 13.84 & 13.02 & 0.56 & 1.09 & $\mathrm{SAB}(\mathrm{rs}) \mathrm{s}$ & 6025 \\
\hline CPG575a & NGC7469 & $230046+0836.9$ & 12.86 & 12.65 & 12.56 & 0.21 & 0.29 & $\mathrm{SAB}(\mathrm{rs}) \mathrm{s}$ & 5083 \\
\hline CPG575b & IC5283 & $230046+0836.9$ & 13.72 & 12.98 & 11.71 & 0.74 & 1.27 & $\mathrm{SA}(\mathrm{r}) \mathrm{cdpec}$ & 5220 \\
\hline CPG577a & CGCG406-013 & $230818+0852.0$ & 14.92 & 14.18 & 12.95 & 0.74 & 1.23 & $\mathrm{E}$ & 11646 \\
\hline CPG577b & CGCG406-013 & $230818+0852.0$ & 14.94 & 14.01 & 12.74 & 0.93 & 1.27 & $\mathrm{E} \rightarrow \mathrm{S} 0 ?$ & 11762 \\
\hline CPG580a & NGC7587 & $231524+0923.5$ & 13.35 & 12.06 & 10.92 & 1.29 & 1.14 & SBab & 8819 \\
\hline CPG580b & CGCG406-051 & $231524+0923.5$ & 14.53 & 14.03 & 12.55 & 0.50 & 1.48 & $\mathrm{~S}$ & 8508 \\
\hline CPG583a & CGCG380-058 & $232400+0202.5$ & 14.46 & 13.54 & 12.23 & 0.92 & 1.31 & $\mathrm{~S} 0 ? \rightarrow \mathrm{S} 0$ & 5876 \\
\hline CPG583b & CGCG380-059 & $232400+0202.5$ & 14.46 & 13.54 & 12.23 & 0.92 & 1.31 & $\mathrm{Sa}$ & 5561 \\
\hline CPG587a & NGC7714 & $233345+0152.7$ & 11.86 & 11.99 & 11.99 & -0.13 & 1.16 & $\mathrm{SB}(\mathrm{s}) \mathrm{b}$ & 2980 \\
\hline CPG587b & NGC7715 & $233345+0152.7$ & 11.81 & 12.14 & 10.22 & -0.33 & 1.92 & Impec & 2933 \\
\hline
\end{tabular}

CPG577 is an early-type pair. No common halo was found. The surface brightness profiles show that CPG577b is a lenticular galaxy. The surface brightness profiles of CPG577a follow an $r^{1 / 4}$ law. The ellipticity of the isophotal contours is quite irregular, varying from $\approx 0.4$ to 0.2 in the inner region between 6 to 10 arcsec, and from $\approx 0.2$ to 0.4 outside 10 arcsec.

CPG580 is a late-type pair with one of the galaxies very disturbed and with a barred spiral with a dust lane and a warped disk (NGC 7587).
CPG583 is a mixed pair, with a spiral and a lenticular without any clear peculiarity.

CPG587 is a late-type pair with two very disturbed late-type galaxies. NGC 7715 has an irregular shape and NGC 7714 has a bar and a peculiar ring.

We have classified these pairs according to the degree of distortion that each galaxy presents. We used a slightly modified version of the classification scheme proposed by Dahari (1985). Pairs with galaxies that showed strong signs of interaction were called $I$ which corresponds 


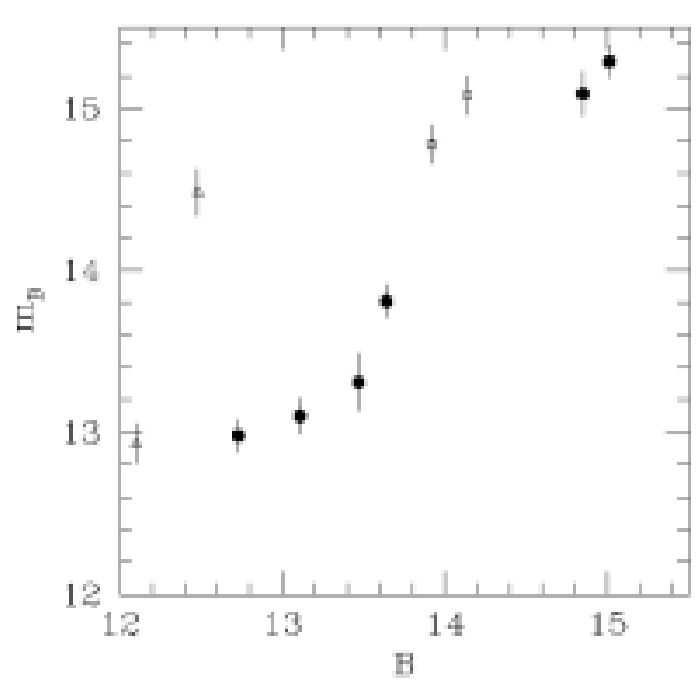

Fig. 6. Comparison between our values of $B$ magnitudes before corrections (galactic extinction and inclination corrections) and $m_{B}$ from RC3. Filled circles correspond to six galaxies that we had in common with RC3 and with magnitude differences $\leq 0.23$. The others symbols correspond to the pair CPG587 (triangles), the galaxy CPG575a (square), and the galaxy CPG580a (star) whose differences related to RC3 are discussed in the text. The error bars are those given in the RC3

to classes 4, 5 and 6 of Dahari. Pairs with moderate signs of interaction were called $M$ (2 and 3 in Dahari's scheme) and pairs with weak or no sign of interaction were called $W$. Pairs that had one galaxy disturbed and one normal were classified as $M-W$. In Table 3 we present the classification of the peculiarities according to this scheme, the angular separation $\left(\theta\left(^{\prime}\right)\right.$ ), the projected separation (in $\mathrm{h}^{-1}$ $\mathrm{kpc}$ ) and the radial velocity difference (in $\mathrm{km} \mathrm{s}^{-1}$ ).

We have looked for star formation regions in the galaxies of our sample. Color maps $(B-V$ and $V-I)$ were used to identify these regions. Blue regions, blue tails, bars, patchy and knotty features were identified in most of the late-type galaxies. The strongest tidal damage and star formation regions are found in CPG083 and CPG551. CPG083 is a mixed pair with late-type galaxy completely disrupted and with prominent blue star formation regions (see also Hippelein 1989). CPG551a presents several blue knots typical of star formation activity and CPG551b presents a blue tail and a blue central bar. We have used color maps in order to identify dust regions and found clear signs of dust in early and late-type galaxies. CPG091b has the strongest equatorial dust lane of our sample. The warped galaxy CPG580a also shows a strong dust feature. Another interesting result is the number of barred galaxies found in our sample. Six late-type galaxies are barred, however no late-type pair was found to have two barred galaxies. Reduzzi \& Rampazzo (1996) also found a frequent presence of bars in pairs and suggested that bars would be an efficient transient mechanism to transfer gas to the nuclear region and produce nuclear activity.

We have also looked for inner substructures in the early-type galaxies in our sample following the same procedure used in de Mello et al. $(1995,1996)$ and Reduzzi et al. (1994). Elliptical models were subtracted from the images and residual images were created using the ELLIPSE task (IRAF). In this procedure the residual intensity along each ellipse are parametrized in terms of harmonic components. The deviations from a pure ellipse are expressed as a Fourier series such that the fourth cosine term $(\mathrm{C} 4)$ describes whether the isophotes are pointy (positive) or boxy (negative). After careful residual images analysis we verify that six early-type galaxies show residual features indicating deviations from elliptical isophotes. On the other hand, for the remaining five early-type galaxies in our sample (CPG018b, CPG083a, CPG099a, CPG547a and CPG548b) no substructure is observed, and the residual images of these galaxies are very similar to E506-01 shown in Reduzzi et al. (1994).

The images of CPG018a, CPG091b and CPG577a, after subtraction, show ripples and asymmetries like AM2312-511 (Reduzzi et al. 1994). The model-subtracted image of CPG099b presents a substructure, like a cross, elongated towards the tidal deformation (south) of the galaxy, similar to the E5720420 (Reduzzi et al. 1996). They classified this residual feature as "discy isophotes". According to Reduzzi et al. (1996b), after model subtraction, discy ellipticals show a filament of light which corresponds to the area where the model underestimates the light. The filament could be real or artificial; the later produced by the combined effects of pointy isophotes and twisting of the isophotes. The calculated average value of the coefficient $\mathrm{C} 4$ between $10^{\prime \prime}$ and $20^{\prime \prime}$ for this galaxy is -0.02 . The residual images of the CPG577b and CPG583a show the most interesting inner features in our sample. CPG577b have inner shells like the residual map of NGC 2300 (Forbes \& Thomson 1992, Plate 2a) and CPG583a shows an X-structure similar to the E556-130 (Reduzzi et al. 1994). Deviations, such as X-structures, could be associated to the fact that we were adjusting an elliptical model to a boxy galaxy. Reduzzi et al. (1996b) concluded that in E556-130 (NGC 2211), the X-structure is described by sudden variation in the trend of $\mathrm{C} 4$ from a boxiness of up to -1.6 percent to a diskiness which reaches 2 percent. However, Forbes \& Thomson (1992) have suggested that a variety of phenomena could be responsible for the boxy isophotes. We verified a similar variation of $\mathrm{C} 4$ as a function of semi-major axis for both CPG577b $\left(-0.007\right.$ inner $15^{\prime \prime}$ to 0.02 out) and CPG583a (-0.01 inner $12^{\prime \prime}$ to 0.03 out).

\section{Discussion}

The colors of pairs members follow the so-called Holmberg effect which is a correlation between the colors of the 
Table 3. Morphologies

\begin{tabular}{cllccr}
\hline Name & Type & Int.Class & $\begin{array}{c}\theta \\
\left({ }^{\prime}\right)\end{array}$ & $\begin{array}{c}\text { Sep. } \\
\left(\mathrm{h}^{-1} \mathrm{kpc}\right)\end{array}$ & $\begin{array}{c}\Delta V \\
\left(\mathrm{~km} \mathrm{~s}^{-1}\right)\end{array}$ \\
\hline & & & & \\
CPG 018 & EE & $M$ & 0.34 & 18.6 & 355 \\
CPG 069 & SS & $I$ & 0.70 & 16.9 & 37 \\
CPG 083 & ES & $I$ & 0.71 & 17.8 & 200 \\
CPG 091 & ES & $M-W$ & 0.72 & 22.8 & 412 \\
CPG 099 & EE & $I$ & 0.97 & 10.3 & 305 \\
CPG 545 & SS & $W$ & 0.34 & 05.7 & 30 \\
CPG 547 & ES & $I$ & 0.67 & 15.3 & 31 \\
CPG 548 & ES & $M-W$ & 1.76 & 21.6 & 387 \\
CPG 551 & SS & $I$ & 0.98 & 17.2 & 8 \\
CPG 575 & SS & $I$ & 1.32 & 19.8 & 137 \\
CPG 577 & EE & $W$ & 0.47 & 16.0 & 116 \\
CPG 580 & SS & $I$ & 0.82 & 20.7 & 311 \\
CPG 583 & ES & $W$ & 1.00 & 16.6 & 315 \\
CPG 587 & SS & $I$ & 1.88 & 16.2 & 47 \\
& & & & & \\
\hline
\end{tabular}

pairs members (Holmberg 1954; Demin et al. 1984). In Fig. 7 we show the $(B-V)$ plot for each component $(a, b)$ of the pairs. Late-type pairs are marked as filled circles, early-type pairs as open circles and mixed-pairs as stars. Reduzzi \& Rampazzo (1995) using a more complete sample found that while the colors of the members correlate significantly for EE and SS pairs, the correlation for ES is less significant. It suggests that pairs of different morphologies should be analyzed differently. In pairs with two gas-rich components (SS) tidal forces perturb the gas and the stars over the entire disk causing severe damage to both galaxies. SS pairs such as CPG551 and CPG587 have strong tails probably caused by the slow encounter between the galaxies. Other SS pairs have barred galaxies probably also created by tidal effects. However, our sample is too small to allow any statistical analysis.

Four of our pairs are now confirmed to have a lenticular galaxy as a component. Rampazzo et al. (1992 and 1995) had also found an excess of lenticulars in their sample. In fact, they had estimated that $10-15 \%$ of all pairs classified as mixed pairs would in fact be disky pairs, i.e. formed by a spiral galaxy and a lenticular galaxy. This result suggests that S0's could be the result of interaction. Charlton et al. (1992) have proposed a toy-model for evolution of morphological types in a merger scenario which also agrees with our results. They have considered that mergers between unequal mass galaxies make S0's and mergers between equal mass galaxies make ellipticals. In their model S-S0 pairs are formed from an initial group of 3-5 galaxies.

The distortion on the isophotal shapes of elliptical galaxies are frequently used in studies of environmental

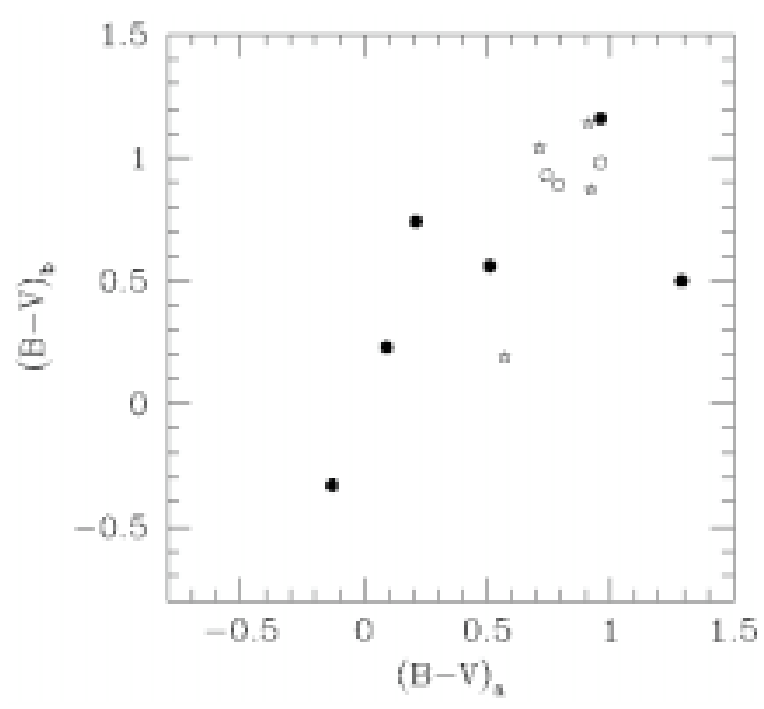

Fig. 7. $B-V$ plot for each component $(a, b)$. Filled circles are late-type pairs, open circles are early-type pairs and stars are mixed-pairs

effects as evidences of tidal effects. For instance, Bender (1988) has suggested that elliptical galaxies can be classified according to their flatness and Bender et al. (1989) suggested that boxy isophotes are signs of past merger (Bender et al. 1989). Caon \& Einasto (1995) have suggested that this property is more sensitive to the environment rather than the bulge/disk ratio. They found that disky elliptical galaxies are located in lower density environments and boxy ellipticals in higher densities. However, more recently, Andreon (1996) using a more complete sample of galaxies in the Coma cluster has suggested that diskiness and boxiness has no environmental origin. However, because our sample of galaxies is made of isolated pairs and that galaxies in clusters are more influenced by the cluster as a whole than by neighboring galaxies (Makino \& Hut 1997) it is possible that tidal effects on the internal properties of galaxies be more effective in pairs than in clusters. In this study we verified that 6 galaxies show deviations from their elliptical isophotes or inner substructures; the most interesting results were obtained for $2 \mathrm{~S} 0$ galaxies. One of them, CPG577b, shows inner shells and the other one, CPG583a, exhibits X-structures. However, because of the small size of our sample we are unable to draw any conclusion on the environmental origin of these features. However, our results agree with previous ones and show that X-structures is one of the less frequently observed forms of fine structure (less than $10 \%$ of our sample show this structure).

It is well known that interaction can trigger both thermal and non-thermal activity, i.e. starburst and AGN (Dahari 1985; Gallimore \& Keel 1993; Keel \& van Soest 1992; Liu \& Kennicutt 1995). However, while many 
active galaxies show signs of interactions, not all interacting galaxies show signs of activity. An intuitive solution for this dichotomy is the fact that tidal damage is longlived (few Gyr) while any form of activity is short lived $\left(10^{8} \mathrm{yr}\right)$. Keel et al. (1985) showed that Seyfert activity is over-represented in early stage interactions. Liu \& Kennicutt (1995) work on merging systems found a very large range of spectral properties, ranging from completely evolved stellar populations to starburst and post-starburst systems. However, samples of galaxies such as the one selected by Bergvall \& Johansson (1995) lack of such effects (N. Bergvall private communication). This is may be due to the fact that Bergvall \& Johansson's sample includes weak cases of interaction and pairs with large separations, which is particularly different from the sample that we are analyzing. Some pairs of galaxies of our sample are in stages of interaction which present tidal damage and also activity. There is strong tidal damage on late-type galaxies such as CPG083, CPG551, CPG580 and even on early-type galaxies such as CPG099. There is Seyfert activity in CPG083 and CPG575 (Hippelein 1989 and Marquez \& Moles 1994) and several star formation regions in CPG551a and CPG580b. However, one has to take into account that pairs of different morphologies may have passed to different evolution processes and therefore show different effects. Moreover, activities in disturbed disks have a longer lifetime due to a larger available gas supply in a disturbed disk (Keel 1996).

\section{Conclusions}

We have selected a local sample of pairs of galaxies with the aim of building a photometric database which will be used in the future for comparing local pairs properties with more distant pairs. A few properties of local pairs that we have discussed in this paper are:

1) galaxies in close pairs are in general bluer than isolated galaxies,

2) close pairs of galaxies have an excess of early-type galaxies.

3) Late-type pairs show strong tidal damage to their morphology and blue star formation regions,

4) many elliptical galaxies in close pairs are in fact lenticular galaxies (2 lenticular galaxies have blue star formation regions),

5) pairs with smaller radial velocity difference present stronger signs of interaction,

6) pairs can present activity and tidal damage simultaneosly.

Moreover, one should take into account that local pairs of different morphology may have passed to a different evolution process which violently transformed their morphology. Pairs with at least one early-type component may be descendents of groups of galaxies, i.e. the early-type galaxy been already a result of a collapse of a group of galaxies. However, late-type pairs are probably long-lived showing clearly signs of interaction. Some of them could be seen as an early stage of mergers because they are still separated but with their disks very disturbed. One has also to account for the fact that more distant pairs could be in the process of formation and would have different properties when compared to these local pairs.

Acknowledgements. SJ and DFM thank CNPq for the fellowship and LI thanks Fondecyt Chile for support through "Proyeto FONDECyT 8970009" and from a 1995 Presidential Chair in Science. We are grateful to M.V. Alonso, N. Bergvall, W.C. Keel and J.M. Schwartzenberg for helpful comments and W. Freudling for providing GALPHOT. DFM thanks the hospitality at Universidad Catolica and ESO, Chile, and UNESCO for the international grant.

\section{References}

Andreon S., 1996, A\&A 314, 763

Barnes J.E., Hernquist L., 1992, ARA\&A 30, 705

Barnes J.E., Hernquist L., 1996, ApJ 471, 115

Baugh C.M., Cole S., Frenk C.S., 1996, MNRAS 283, 1361

Bender R., 1988, A\&A 193, L7

Bender R., et al., 1989, A\&A 217, 35

Bergvall N., Johansson L., 1995, A\&AS 113, 499

Binggeli B., 1980, A\&A 82, 289

Binney J., Tremaine S., 1987, Galactic Dynamics. Princeton Univ. Press, Princeton, NJ

Borne K.D., Hoessell J.G., 1988, ApJ 330, 51

Boroson T., 1981, ApJS 46, 177

Bower R.G., Lucey J.R., Ellis R., 1992, MNRAS 254, 601

Burstein D., 1979, ApJ 234, 435

Burstein D., Heiles C., 1984, ApJS 54, 33

Buta R., Crocker D.A., 1993, AJ 105, 1334

Butcher H., Oemler A., 1978, ApJ 219, 18

Butcher H., Oemler A., 1984, ApJ 285, 426

Caon N., Einasto M., 1995, MNRAS 273, 913

Carlberg R.G., Pritchet C.J., Infante L., 1994, ApJ 435, 540

Charlton J.C., Whitmore B., Gilmore D.M., 1992, in "Groups of Galaxies", ASP Conf. Ser. 70, Ritcher O.G. \& Borne K. (eds.)

Dahari O., 1985, ApJS 57, 643

de Mello D.F., Keel W.C., Sulentic J.W., et al., 1995, A\&A 297, 331

de Mello D.F., Sulentic J.W., Reduzzi L., Rampazzo R., 1996, A\&A 308, 387

de Mello D.F., Infante L., Menanteau F., 1997a, ApJS 108, 99 de Mello D.F., Infante L., Menanteau F., Vieira G., 1997b, ApJS 110, 227

Demin V., Zasov A., Dibai E., Tomov A., 1984, Soviet Astron. 28,367

de Souza R.E., de Mello D.F., dos Anjos S., 1997, A\&AS (in press)

de Vaucouleurs G., 1948, A\&A 11, 247

de Vaucouleurs G., de Vaucouleurs A., Corwin H.G., 1976, Second Reference Catalog of Bright Galaxies. Austin: University of Texas Press, (RC2)

de Vaucouleurs G., de Vaucouleurs A., Corwin H.G., et al., 1991, Third Reference Catalog of Bright Galaxies. Springer, New York, (RC3)

Forbes D.A., Thomson R.C., 1992, MNRAS 254, 723 
Fritze-v. Alvensleben U., 1996, in "From Stars to Galaxies: The Impact of Stellar Physics on Galaxy Evolution" Leitherer C., Fritze-v. Alvensleben U. and Huchra J. (eds.), ASP Conf. Ser. 98, 496

Franx M., Illingworth G., de Zeeuw T., 1991, ApJ 383, 112

Gallimore J.F., Keel W.C., 1993, AJ 106, 1337

Héraudeau Ph., Simien F., 1996, A\&AS 118, 111

Hippelein H.H., 1989, A\&A 216, 11

Holmberg E., 1937, Ann. Lunds Astr. Obs., 6

Holmberg E., 1954, Medd. Lunds Astr. Obs., 186

Holmberg E., 1958, Medd. Lunds Astr. Obs. Ser. II, 136, 1

Hubble E., 1926, ApJ 64, 321

Hubble E., 1936, in "The Realm of the Nebulae", New Haven. Yale Univ. Press

Infante L., de Mello D.F., Menanteau F., 1996, ApJ 469, L85

Jedrzejewski R.I., 1987, MNRAS 226, 747

Junqueira S., de Freitas Pacheco J.A., 1994, AJ 108, 1627

Kailey W.F., Lebofsky M.J., 1988, ApJ 326, 653

Karachentsev I., 1972, Comm. Spec. Astrophys. Obs. 7, 1

Keel W.C., Kennicutt R.C., Hummel E., van der Hulst J.M., 1985, AJ 90, 708

Keel W.C., van Soest E.T.M., 1992, A\&AS 94, 553

Keel W.C., 1993, AJ 106, 1771

Keel W.C., 1996, AJ 111, 696

Landolt A.U., 1992, AJ 104, 340

Liu C.T., Kennicutt R.C., 1995, ApJS 100, 325

Makino J., Hut P., 1997, ApJ 481, 83

Marquez I., Moles M., 1994, AJ 108, 90 (MM)

Marquez I., Moles M., 1996, A\&AS 120, 1 (MM)

Moore B., Katz N., Lake G., 1996, in "New Light on Galaxy Evolution" IAU Symposium 171, Bender R. \& Davies R.L. (eds.). Kluwer Publishers

Noguchi M., 1988, A\&A 203, 259

Page T., 1952, ApJ 116, 63

Page T., 1961, AJ 66, 61

Rampazzo R., Sulentic J.W., 1992, A\&A 259, 43

Rampazzo R., Reduzzi L., Sulentic J.W., Madejsky R., 1995, A\&AS 110, 131

Reshetnikov V.P., Hagen-Thorn V.A., Yakovleva V.A., 1993, A\&AS 99, 257

Reduzzi L., Rampazzo R., Sulentic J.W., Prugniel P., 1994, The Messenger 75, 28

Reduzzi L., Rampazzo R., 1995, Astroph. Lett. Comm. 30, 1

Reduzzi L., Rampazzo R., 1996, A\&AS 116, 571

Reduzzi L., Longhetti M., Rampazzo R., 1996, MNRAS 282, 149

Schombert J.M., Bothun G.D., 1987, AJ 93, 61

Schweizer F., 1983, in "Internal Kinematics \& Dynamics of Galaxies" IAU Symposium 100, Athanassoula E., Reidel D. (eds.). Dordrecht, p. 319

Soares D.S., de Souza R.E., de Carvalho R.R., Couto da Silva T.C., 1995, A\&AS 110, 371

Sulentic J.W., 1992, in "Morphological and Physical Classification of Galaxies", Longo G., Capaccioli M. and Busarello G. (eds.). Kluwer, p. 293

Toomre A., Toomre J., 1972, ApJ 178, 623

Toomre A., 1977, in "The Evolution of Galaxies and Stellar Populations" Tinsley B.M. and Larson R.B. (eds.). New Haven: Yale University, p. 401

Williams R., et al., 1996, AJ 112, 1335

Zepf S.E., Whitmore B.C., 1993, ApJ 418, 72 